\title{
Relationship between magnitude of body weight effects and exposure duration in mammalian toxicology studies and implications for ecotoxicological risk assessment
}

Magnus Wang $^{1 *} \mathbb{D}$, Anja Guckland ${ }^{1}$, Roger Murfitt ${ }^{2}$, Markus Ebeling ${ }^{3}$, Dennis Sprenger ${ }^{4}$, Manousos Foudoulakis ${ }^{5}$ and Apostolos Koutsaftis ${ }^{6}$

\begin{abstract}
Background: For regulatory approval of pesticides in the EU, an ecotoxicological risk assessment has to be conducted including an assessment of long-term effects on mammals. For this assessment, toxicity studies are considered which are conducted with rodents which are continuously exposed via diet over a long period. A typical observation in these studies is a reduction of body weight. Such reductions are generally more pronounced at the end of a study and are often used to derive an endpoint for the wild mammal long-term risk assessment. However, exposure in the field is rather short for most modern pesticides. Therefore, the change of the magnitude of effects over exposure time may be relevant to obtain a realistic view of effects expected in the field.

Results: Time dependence of effects observed in toxicity studies conducted with rats was evaluated, focusing on effects on female body weight. Benchmark doses $\left(\mathrm{BMD}_{10}\right.$, i.e., $10 \%$ effect) were calculated for a total of 37 long-term toxicity studies conducted with 13 different active substances used as pesticides. Female body weights after 14, 21, 28,42 and 70 days of dosing were used for BMD analysis per active substance to evaluate time-dependent changes of $\mathrm{BMD}_{10}$. $\mathrm{BMD}_{10}$ values declined continuously with exposure duration, indicating that the longer the duration of exposure, the greater are the effects on body weights. This continuous decline was observed for all pesticide classes (i.e. herbicides, insecticides and fungicides) from the studies analyzed. After 70 days, the $B M D_{10}$ levels were about half of the $\mathrm{BMD}_{10}$ at day 14 .

Conclusion: The results indicate that animals respond to pesticide exposure in an exposure-time-dependent way, i.e. effects on body weight of the animals are less pronounced when the duration of exposure is short. The greatest body weight effects were observed at the end of toxicity studies (after longest exposure). The realism of the current wild mammal risk assessment for plant protection products is discussed and how it could be improved by considering an appropriate time period for the selection of endpoints in chronic toxicity studies, which reflects the exposure time of free ranging animals in the field.
\end{abstract}

Keywords: Benchmark dose, Risk assessment, Time dependence, Rats, Body weight

\footnotetext{
*Correspondence: magnus.wang@wsc-regexperts.com

${ }^{1}$ WSC Scientific GmbH, Dossenheimer Landstr. 9/1,69121 Heidelberg, Germany

Full list of author information is available at the end of the article
} 


\section{Background}

For regulatory approval of pesticides in the EU, an ecotoxicological risk assessment has to be conducted, which includes an assessment of long-term effects on mammals. For this assessment, a number of toxicity studies with rodents (conducted in order to determine safety to humans) are considered to determine the potential longterm toxicity of pesticides to wild mammals [3]. One key study here is the rat multi-generation reproduction study, conducted according to OECD Test Guideline 416 [8]. In that study, the animals are treated for 10 weeks in the so-called "pre-mating phase" before the actual reproduction phase is initiated. Also in other potentially relevant studies (e.g., the 90-day study following OECD 408, or the chronic study following OECD 452/453), the animals are continuously exposed via diet over a long period. At higher doses, a typical observation in many of these studies is a reduction of body weight, which is generally more pronounced at the end of a study. At present, the maximum reduction of body weight observed at the end of studies is usually used to estimate an endpoint for use in determining potential risk to wild mammals. However, since body weight reduction is generally most pronounced at the end of such studies, the question arises to what extent exposure duration determines the strength of effect and how this might be of relevance for the risk assessment. This is of particular importance, since the exposure in the field is rather short for most pesticides (according to EFSA [3], a default $\mathrm{DT}_{50}$ of 10 days is considered as a worst case, with calculation of a weighted average concentration over 21 days). Hence, the change of the magnitude of effects over exposure time may be relevant to obtain a realistic view of effects expected in the field.

The principle that sublethal effects typically become stronger with exposure time is a general phenomenon that has previously been described in rodents for pesticides, pharmaceuticals and heavy metals $[6,7,9,13]$. Furthermore, mortality has been shown to depend on exposure time as well (e.g. [14]) and an exposure time dependence of toxicity has also been described for aquatic organisms (e.g. [2, 10, 12]). Hence, the exposure time dependence is an important and so far often neglected aspect of the regulatory ecotoxicological risk assessment, in particular for the long-term assessment. A systematic, comprehensive analysis of the time dependence of long-term toxicity, including a large number of substances and several time points has not been conducted previously. Therefore, a meta-analysis was performed considering 37 long-term toxicity studies conducted on rats with 13 different pesticidal substances. As an example, the parameter female body weight was considered and benchmark doses $\left(\mathrm{BMD}_{10}\right.$; estimation of the dose which causes $10 \%$ effect based on dose-response data) were calculated. A Benchmark response of $10 \%$ was selected, corresponding to the $\mathrm{EC}_{10}$ currently required in the EU regulation. Benchmark dose calculations were conducted according to the report of the EFSA Scientific Committee [4].

\section{Materials and methods \\ Data description}

Long-term toxicity studies on rats were collected for 13 pesticides (Table 1). To increase the scope and robustness of the evaluation, the analysis included not only multigeneration reproduction studies, but also studies for testing sub-chronic oral toxicity, chronic oral toxicity (partly including carcinogenicity testing) and sub-chronic neurotoxicity, which all include a similar test design; i.e. start with young adult rats which are continuously administered the test substance via the diet. Therefore, due to the similar test design, the data from the initial 10 weeks from all these study types were used for further analysis where available. Only those substances were selected which caused a dose-dependent decrease of at least $10-20 \%$ in female body weight (female body weight was considered because females are more relevant for wild mammal population growth than males). Substances were only included when at least two suitable studies per substance were available. The maximum was four suitable studies for one substance.

Furthermore, substances were chosen to represent a variety of modes of action classes and pesticide classes (fungicides, herbicides and insecticides). Five of the

Table 1 List of compounds for which toxicity studies were evaluated

\begin{tabular}{llll}
\hline Common name & Pesticide class & Mode of action & CAS no. \\
\hline Fenpropidin & Fungicide & FRAC G2 & $67306-00-7$ \\
Fenpropimorph & Fungicide & FRAC G2 & $67564-91-4$ \\
Fludioxonil & Fungicide & FRAC E2 & $131341-86-1$ \\
Propineb & Fungicide & FRAC "multi-site & $12071-83-9$ \\
& & Contact activity" & \\
Bromoxynil & Herbicide & HRAC C3 & $1689-84-5$ \\
Isoproturon & Herbicide & HRAC C2 & $34123-59-6$ \\
Propyzamide & Herbicide & HRAC K1 & $23950-58-5$ \\
Prosulfuron & Herbicide & HRAC B & $94125-34-5$ \\
Pyrazone & Herbicide & HRAC C1 & $1698-60-8$ \\
Imidacloprid & Insecticide & IRAC 4A & $105827-78-9$ \\
Methiocarb & Insecticide & IRAC 1A & $2032-65-7$ \\
MK-239 & Insecticide & IRAC 21A & $119168-77-3$ \\
Pymetrozine & Insecticide & IRAC 9B & $123312-89-0$ \\
\hline
\end{tabular}

Mode of action refers to RAC classes (IRAC (insecticides): https://www.irac-onlin e.org, FRAC (fungicides): http://www.frac.info, HRAC (herbicides): https://www. hracglobal.com) 
selected substances were herbicides (HRAC codes: B, C1, C3, K1), four were fungicides (FRAC codes: E2, G2 and "multi-site contact activity") and four were insecticides (IRAC codes: 1A, 4A, 9B, 21A, see Table 1).

From these toxicity studies, the female body weight data at days $14,21,28,42$ and 70 ( \pm 2 days) were selected. The achieved doses were calculated for the period from study start until the respective day of body weight measurement. The data from all studies for a substance were pooled for BMD analysis using the relative body weight of each dose group (normalized to \% of the body weight of the respective concurrent control).

\section{Selection of the benchmark response (BMR)}

The data considered here were provided as continuous data for groups of animals. The default BMR as proposed by EFSA Scientific Committee [4] for continuous data is $5 \%$ (change of mean response). However, according to Commission Regulation (EU) No 283/2013 [1], the $\mathrm{EC}_{10}$ and $\mathrm{EC}_{20}$ are relevant endpoints for the ecotoxicological risk assessment; in addition, the $\mathrm{EC}_{5}$ can often not be reliably estimated since it is very near to the No Effect Level. Therefore, the $\mathrm{BMD}_{10}$ and $\mathrm{BMD}_{20}$ were considered relevant for this analysis. A first review of the data revealed that the $20 \%$ effect level is rarely reached in any of the studies; therefore, $\mathrm{BMD}_{20}$ could not be reliably estimated and as a consequence, it was decided to focus on a benchmark response (BMR) of $10 \%$ effect level $\left(\mathrm{BMD}_{10}\right)$.

\section{Software}

Benchmark dose (BMD) calculations were performed according to EFSA Scientific Committee [4] using the R library PROAST (version 62.10), developed by the National Institute for Public Health and the Environment (RIVM) of the Netherlands.

\section{Selection of dose-response models}

According to EFSA Scientific Committee [4], different dose-response models are tested to find all plausible BMD values. The models recommended by EFSA Scientific Committee [4] for continuous data are the 3-parameter model (called Model 3 in PROAST) and the 4-parameter model (called Model 5 in PROAST) of the Hill and the Exponential family. Additionally, the Nullmodel and the Full-model should be fitted.

The Model 5 (4-parameter model) was not used in this analysis because a preliminary evaluation has shown that fits with this model often resulted in very unrealistic dose-response curves [5]. For example, for one substance (fungicide) the fit for day 14 indicates that the maximum benchmark response (BMR) is only about 10\% (Fig. 1a). This is biologically not reasonable, since reduction of body weight can be considerably larger than $10 \%$, i.e. there is no natural limit of body weight reduction at this level. This can be seen from the results for other substances. There will rather be a cut-off due to mortality when body weight decreased by a much greater amount. Therefore, for endpoints such as body weight, it is not suitable to consider all available models, but to select the biologically reasonable ones (see Fig. 1b).

\section{Calculation of confidence intervals}

BMD and the lower (BMDL) and upper (BMDU) confidence intervals are calculated for all models. The BMD/ $\mathrm{BMDL} / \mathrm{BMDU}$ analysis results in a range of BMD and BMDL/BMDU values. To establish the BMD confidence interval and BMDL for the dose-response dataset of the endpoint female body weight, the process described by EFSA Scientific Committee [4] was followed.

\section{Handling of alerts}

An 'alert' occurs when the Akaike information criterion (AIC) of the selected model differs by more than two units from the AIC of the full model. If an alert occurred, it was evaluated whether the difference between the AICs was only minor (e.g. up to three units) or not, and the fit was visually checked. If the difference of the AICs was only minor and the visual fit was acceptable, then the fit and BMD calculations were accepted. Otherwise, if several data points did not match the fit, it was checked if they were derived from the same study or not. If such systematic deviations of responses from the fit occurred, then the BMD calculation was conducted using the variable 'study code' as covariate, to identify if the study differed from the other studies. If one study differed from other studies, it was excluded from the analysis. If deviations of responses were not systematic, i.e. were not caused by one single study, then it was decided by visual inspection if the fit was acceptable or if any data points should be excluded (after evaluating the study reports for any reasons of the deviation in responses).

\section{Results}

Benchmark doses $\left(\mathrm{BMD}_{10}\right)$ and their confidence limits (lower limit $\mathrm{BMDL}_{10}$, upper limit $\mathrm{BMDU}_{10}$ ) were calculated from body weight changes in female rats observed in 37 long-term toxicity studies conducted with 13 different active substances.

Overall, all $\mathrm{BMD}_{10}$ values (and confidence intervals) for all pesticide classes (fungicides, herbicides and insecticides) declined over time (Fig. 2). To further analyze the time dependency of body weight effects after exposure to pesticides, the $\mathrm{BMD}_{10}$ values for days $21,28,42$ and 70 were compared to the 14 -day $\mathrm{BMD}_{10}$ (which was the earliest time point at which the $\mathrm{BMD}_{10}$ was calculated in 


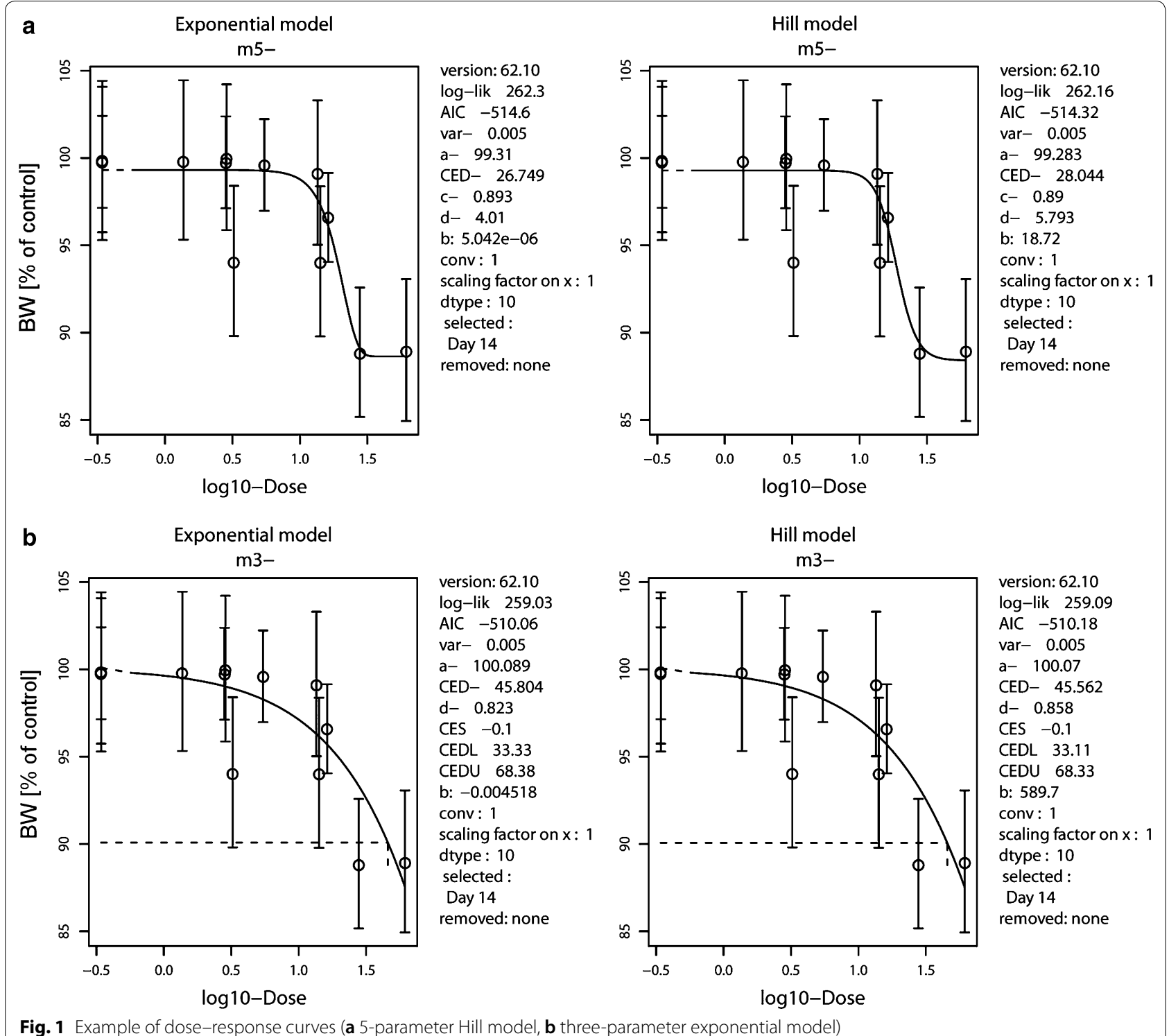

this analysis), i.e. the $\mathrm{BMD}_{10}$ at days $21,28,42$ and 70 was related to the weight at day 14 (which was set to $100 \%$ ). The results were similar for all pesticide classes and were, therefore, summarized (Fig. 3).

\section{Discussion}

After 70 days of exposure, the $\mathrm{BMD}_{10}$ decreased to about half of the value calculated for 14 exposure days. This is a relevant finding for the wild mammal risk assessment, since it indicates that animals respond to pesticide exposure in a clear time-dependent way, showing less pronounced body weight effects when the duration of exposure is short. The strongest body weight effects are usually observed at the end of toxicity studies, while the default time period relevant for the long-term risk assessment is much shorter than the duration of a study. In a typical two-generation rat reproduction study, the females may continuously be exposed for up to 4 months. Long-term risk assessments are usually conducted considering a default exposure period of 21 days. Thus, there is a marked discrepancy when using endpoints based on maximum body weight effects for the risk assessment. For example, the $\mathrm{BMD}_{10}$ at day 21 was $158 \%, 182 \%$ and $145 \%$ of the $\mathrm{BMD}_{10}$ at day 70 for fungicides, herbicides and insecticides, respectively (Table 2). Therefore, using endpoints determined after 70 days of exposure adds a considerable additional margin of safety to the risk 


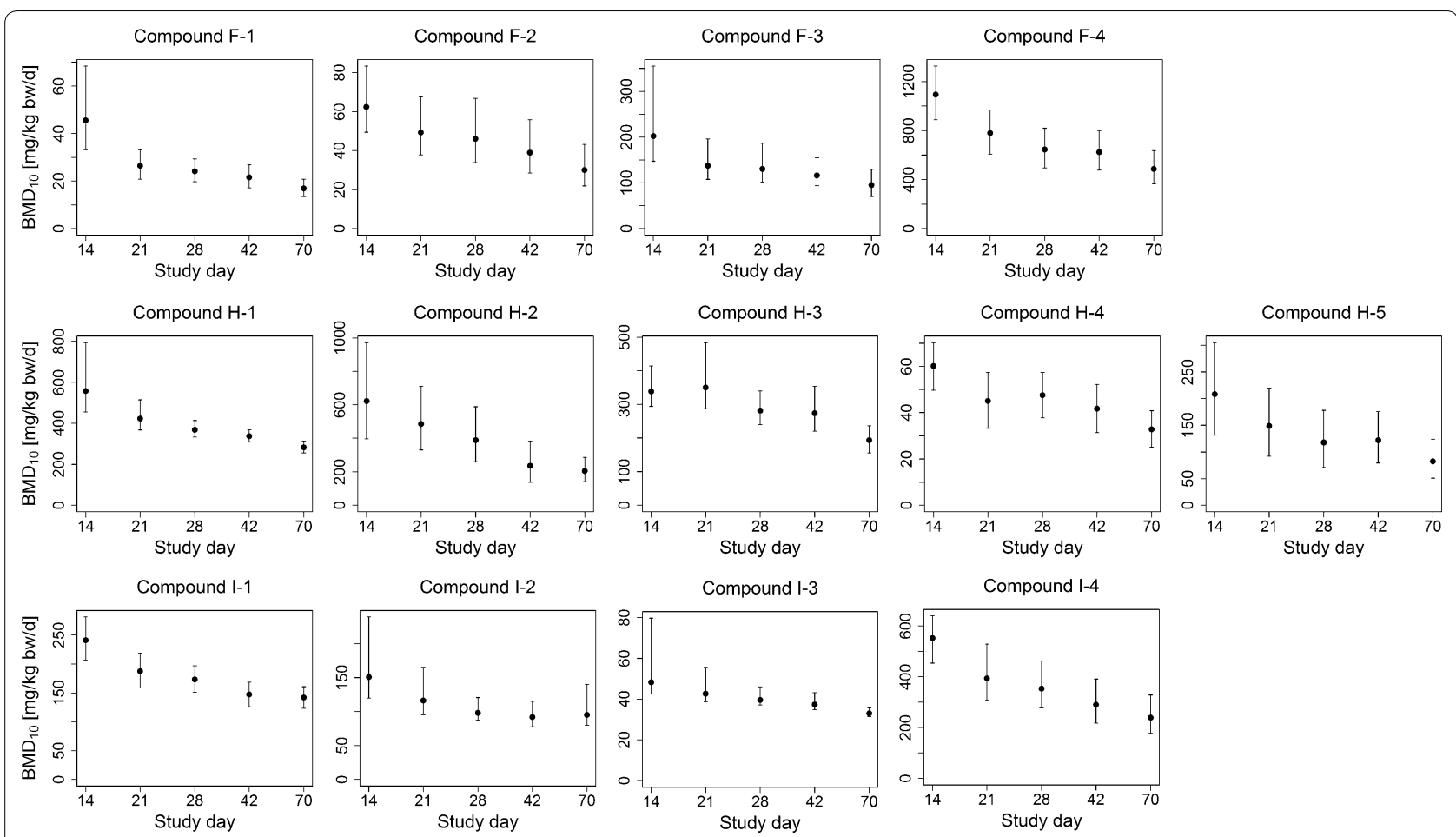

Fig. $2 \mathrm{BMD}_{10}$ values for each substance after 14, 21, 28, 42 and 70 days of exposure. Error bars indicate the upper and lower confidence limits of $\mathrm{BMD}_{10}$

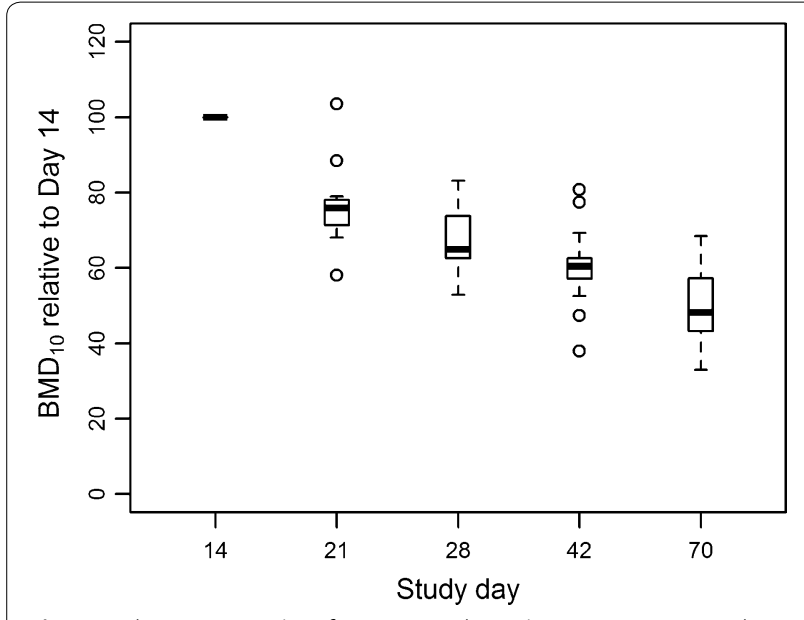

Fig. 3 Relative $\mathrm{BMD}_{10}\left(\%\right.$ of $\mathrm{BMD}_{10}$ on day 14 ) over $21,28,42$ and 70 days (thick line $=$ median over all substances; upper/lower margin of boxes $=$ upper/lower quartile of data; upper/lower whisker = upper/lower values within 1.5-fold of the interquartile range; points $=$ outlier)

assessment. To obtain a more realistic view, an endpoint should be selected which reflects the actual exposure window under field conditions (e.g. 21 days).
Overall, in this meta-analysis, effects increased continuously over time for all pesticide classes (Table 2) and the use of an endpoint which is based on the final body weight reduction provides an overly conservative worst case in the risk assessment. Instead, calculation of the toxicity endpoint for the time period that is relevant for the risk assessment (i.e. the actual exposure time) would provide a more realistic scenario.

In toxicological studies, which are used for ecological risk assessment, the duration of exposure is fixed and not adjusted to the expected exposure duration in the field. However, time dependency is a typical feature of toxicity: the longer the exposure, the greater are the effects (e.g. [11, 14]). This time dependency of toxicity is so far not considered in the ecotoxicological risk assessment. The factor time is rather described as a constant, such as acute, sub-chronic or chronic. For the chronic human consumer risk assessment, the exposure time may not be of high relevance, since consumer exposure is continuous and fairly constant in magnitude, because consumers may eat freshly harvested foodstuff which will always carry similar residue levels. However, for animals in the field, exposure differs fundamentally from consumer exposure, since animals living in a crop are exposed at or shortly after application of a pesticide. Hence, the 
Table 2 Relative $\mathrm{BMD}_{10}$ for body weight reduction of female rats for different exposure durations

\begin{tabular}{lllll}
\hline $\begin{array}{l}\text { Exposure } \\
\text { duration } \\
\text { [days] }\end{array}$ & \multicolumn{4}{l}{$\mathbf{B M D}_{\mathbf{1 0}}\left[\%\right.$ of day $\mathbf{7 0} \mathrm{BMD}_{\mathbf{1 0}}$ ] } \\
\cline { 2 - 5 } & $\begin{array}{l}\text { Fungicides } \\
\mathbf{( \% )}\end{array}$ & $\begin{array}{l}\text { Herbicides } \\
(\%)\end{array}$ & $\begin{array}{l}\text { Insecticides } \\
\mathbf{( \% )}\end{array}$ & Overall (\%) \\
\hline 14 & 223 & 224 & 195 & 216 \\
21 & 158 & 182 & 145 & 164 \\
28 & 135 & 151 & 130 & 140 \\
42 & 127 & 127 & 111 & 123 \\
70 & 100 & 100 & 100 & 100 \\
\hline
\end{tabular}

exposure scenario in chronic toxicity studies (with continuous exposure over months) differs greatly from exposure patterns in the field. This discrepancy is currently not yet addressed explicitly in the ecotoxicological risk assessment. Such an assessment can be done readily, since the information on exposure time and related effects are generally well described in the toxicity studies. In the present meta-analysis, covering 13 pesticides, we found that effects were clearly time dependent. This meta-analysis, which focused on female body weight as a commonly affected endpoint in chronic toxicity studies, is the first focusing explicitly on the time dependence of chronic effects over several substances. However, studies on specific substances (pesticides, pharmaceuticals, heavy metals) already indicated a correlation of the strength of effects and the exposure time $[6,7,9,10$, $13-15]$.

In terms of the ecological risk assessment, the time dependency of effects indicated that the use of effects at the end of a chronic toxicity study (e.g. the final body weight reduction) as endpoint provides an overly conservative worst case for the risk assessment. For example, considering a multi-generation rat study, female bodyweight at the end of lactation may represent effects observed after about 3-4 months of continuous exposure. The realism of the ecotoxicological risk assessment might, therefore, be increased by taking the time dependency of such a toxicological effect into account and choosing an endpoint which reflects the actual exposure duration in the field. This would not necessarily mean that the risk assessment is less protective than it is when choosing an endpoint at the end of a study. On the contrary, one might find that the most relevant effect (e.g. body weight, litter size, etc.) is not the one observed at the end of a study, but another one, which may not be the strongest one at the end of the study, but the strongest during the actual exposure period in the field (e.g. 3 weeks, as assumed by default by EFSA [3]). Hence, considering the actual exposure period for selection of the endpoint for the ecotoxicological risk assessment would reduce uncertainty and increase realism.

\section{Conclusions}

The $\mathrm{BMD}_{10}$ values for all pesticide classes (i.e. fungicides, herbicides and insecticides) decreased continuously with increasing exposure time. This indicates that animals respond to pesticide exposure in a timedependent way, showing less pronounced effects when exposure is short. With increasing duration of exposure to a substance, animals show greater body weight effects.

In the long-term ecotoxicological risk assessment, a time window of 21 days to calculate exposure is considered as a default [3]. However, the present analysis has shown that the $\mathrm{BMD}_{10}$ for this exposure time is always much higher than after 70 days of exposure. Therefore, the use of an endpoint which is based on the final body weight reduction observed in a toxicity study provides an overly conservative worst case in the risk assessment. Instead, calculation of the toxicity endpoint for the time period that is relevant for the risk assessment (exposure time) would provide a realistic worst-case scenario.

\section{Abbreviations}

AIC: Akaike information criterion; BMD: benchmark dose; BMDL: lower confidence limit of the benchmark dose; BMDU: upper confidence limit of the benchmark dose; BMR: benchmark response; $\mathrm{DT}_{50}$ : dissipation half life; $\mathrm{EC}$ : effect concentration; EFSA: European Food Safety Authority; FRAC: Fungicide Resistance Action Committee; HRAC: Herbicide Resistance Action Committee; IRAC: Insecticide Resistance Action Committee; RIVM: National Institute for Public Health and the Environment of the Netherlands.

\section{Acknowledgements}

Data were provided by Adama, BASF, Bayer AG, Corteva Agriscience and Syngenta.

\section{Authors' contributions}

MW and AG conducted all analyses and drafted the manuscript. RM, ME, DS, MF and AK planned the study, selected and interpreted the data and reviewed the manuscript. All authors read and approved the final manuscript.

Funding

The present study was funded by European Crop Protection Agency.

\section{Availability of data and materials}

The datasets analyzed are proprietary and not publicly available but may be available from the corresponding author upon reasonable request.

Ethics approval and consent to participate

Not applicable, since the present study is a re-evaluation of existing data.

\section{Consent for publication}

Not applicable.

\section{Competing interests}

The authors declare that they have no competing interests. RM, ME, DS, MF and AK are employed at companies developing and producing plant protection products. MW and AG work as consultants for these and other clients. 


\section{Author details}

${ }^{1}$ WSC Scientific GmbH, Dossenheimer Landstr. 9/1, 69121 Heidelberg, Germany. ${ }^{2}$ Syngenta, Jealott's Hill International Research Centre, Bracknell, Berkshire RG42 6EY, UK. ${ }^{3}$ Bayer AG, Alfred-Nobelstr. 50, 40789 Monheim am Rhein, Germany. ${ }^{4}$ BASF SE, Speyerer Strasse 2, 67117 Limburgerhof, Germany. ${ }^{5}$ Corteva Agriscience ${ }^{\mathrm{TM}}$, The Agriculture Division of DowDuPont ${ }^{\mathrm{TM}}, 280$ Kifissias $^{\circ}$ Ave. \& 2 Ydras St., 15232 Halandri, Greece ${ }^{6}$ ADAMA Deutschland GmbH, Edmund-Rumpler-Str. 6, 51149 Cologne, Germany.

Received: 25 April 2019 Accepted: 10 June 2019

Published online: 17 June 2019

\section{References}

1. Commission regulation (EU) (2013) Setting out the data requirements for active substances, in accordance with Regulation (EC) No 1107/2009 of the European Parliament and of the Council concerning the placing of plant protection products on the market. J Euro Union 283:1-84

2. Connell DW, Yu QJ, Verma V (2016) Influence of exposure time on toxicity-an overview. Toxicol 355-356:49-53

3. European Food Safety Authority (EFSA) (2009) Guidance document on risk assessment for birds and mammals on request from EFSA. EFSA J 7:1438. https://doi.org/10.2903/j.efsa.2009.1438

4. EFSA Scientific Committee (2017) Update: guidance on the use of the benchmark dose approach in risk assessment. EFSA J 15:4658

5. Guckland A, Wang M, Ebeling M (2017) Experiences with the Draft EFSA (2016) guidance on benchmark dose calculations: reliability of BMD for bird and mammal reproductive risk assessment. In: Poster presented at SETAC Europe 27th annual meeting, Brussels, 7-11 May 2017

6. Kackar R, Srivastava MK, Raizada RB (1997) Induction of gonadal toxicity to male rats after chronic exposure to mancozeb. Ind Health 35:04-111

7. Marques CC, Nunes AC, Pinheiro T, Lopes PA, Santos MC, Viegas-Crespo AM, Ramalhinho MG, Mathias ML (2006) An assessment of time-dependent effects of lead exposure in Algerian mice (Mus spretus) using different methodological approaches. Biol Trace Elem Res 109:75-89
8. Organisation for Economic Co-operation and Development (OECD) (2001) Test No. 416: two-generation reproduction toxicity. In: OECD (ed) OECD guidelines for the testing of chemicals, Section 4. OECD Publishing, Paris. https://doi.org/10.1787/9789264070868-en

9. Rahman MF, Siddiqui MKJ, Jamil K (2000) Acid and alkaline phosphatase activities in a novel phosphorothionate (RPR-11) treated male and female rats. Evidence of dose and time-dependent response. Drug Chem Toxicol 23:497-509

10. Snell TW, Moffat BD, Janssen C, Persoone G (1991) Acute toxicity tests using rotifers. III. Effects of temperature, strain, and exposure time on the sensitivity of Brachionus plicatilis. Environ Toxicol 6:63-75

11. Tennekes HA (2010) The significance of the Druckrey-Küpfmüller equation for risk assessment-the toxicity of neonicotinoid insecticides to arthropods is reinforced by exposure time. Toxicol 280:173-175

12. Tennekes HA, Sánches-Bayo F (2011) Time-dependent toxicity of neonicotinoids and other toxicants: implications for a new approach to risk assessment. J Environ Anal Toxicol S 4:001. https://doi.org/10.4172/21610525.S4-001

13. Trasler JM, Hales BF, Robaire B (1987) A time-course study of chronic paternal cyclophosphamide treatment in rats: effects on pregnancy outcome and the male reproductive and hematologic systems. Biol Reprod 37:317-326

14. Verma V, Yua QJ, Connell DW (2014) Evaluation of effects of long term exposure on lethal toxicity with mammals. Environ Pollut 185:234-239

15. Yu Q, Chaisuksant Y, Connell DW (1999) A model for non-specific toxicity with aquatic organisms over relatively long periods of exposure time. Chemosphere 38:909-918

\section{Publisher's Note}

Springer Nature remains neutral with regard to jurisdictional claims in published maps and institutional affiliations.

\section{Submit your manuscript to a SpringerOpen ${ }^{\circ}$ journal and benefit from:}

- Convenient online submission

- Rigorous peer review

- Open access: articles freely available online

- High visibility within the field

- Retaining the copyright to your article

Submit your next manuscript at $\boldsymbol{\nabla}$ springeropen.com 